\title{
Comparison of the efficacy of Subcision with Fractional Carbon Dioxide Laser versus Subcision with Microneedling Fractional Radiofrequency in the Treatment of Atrophic Post acne Scars
}

\author{
Nabeel K. Al-Hamzawi
}

Department of Dermatology, Al-Diwaniyah Teaching Hospital, Diwaniyah, Iraq

\begin{abstract}
Background: Many interventions have been used to treat post-acne scars, with various degrees of efficacy and adverse reactions. Multimodal approach can achieve better results for improving the physical appearance that even if minor may result in significant distress and reduced quality of life. Objective: To yah, Iraq

compare the efficacy of subcision with fractional carbon dioxide (CO2) laser and subcision with microneedling fractional radiofrequency $(\mathrm{RF})$ in treating post-acne scars and to study their adverse effects. Methods: In this prospective, interventional clinical study, a total of 64 patients with post-acne scars were enrolled. The patients were randomly allocated into two groups of 32 patients each, in which group $A$ was treated with subcision plus fractional carbon dioxide laser (CO2) and group $B$ was treated with subcision plus microneedling fractional radiofrequency (RF) every 6 weeks for a total of four sittings. An objective score was calculated for each patient using the Goodman and Baron scale to evaluate the outcome every visit during treatment and then 3 months after completion of therapy. Results: In group A, the percentage reduction in the mean objective scores was found to be $64 \%$. In group $B$, the percentage reduction in mean objective scores was found to be $42 \%$. Most of the changes were seen in the boxcar and rolling types of scars. Conclusions: Subcision and fractional CO2 laser were considered superior over

*Correspondence to Author:

Nabeel K. Al-Hamzawi

Department of Dermatology, Al-Diwaniyah Teaching Hospital, Diwani-

How to cite this article:

Nabeel K. Al-Hamzawi.Comparison of the efficacy of Subcision with Fractional Carbon Dioxide Laser versus Subcision with Microneedling Fractional Radiofrequency in the Treatment of Atrophic Post acne Scars.American Journal of Dermatological Research and Reviews, 2021, 4:44 subcision with microneedling fractional (RF) technique for the treatment of post-acne scars. Boxcar and rolling types of scars showed a high response to treatment than icepick scars

\section{eScîPPub}

eSciPub LLC, Houston, TX USA. Website: https://escipub.com/
\end{abstract}




\section{Introduction:}

The prevalence of acne vulgaris is over $90 \%$ among adolescents and persists into adulthood in approximately $12 \%$ of cases with high psychological and social implications $\left[^{[1,2]}\right.$.

The pathogenesis of acne is multifactorial, including increased sebum production, androgen activity, the proliferation of Propionibacterium acnes ( $P$. acnes) within the follicle, and follicular hyperkeratinization ${ }^{[3]}$. The previous inflammatory process may result in tissue injury that creates scar formation.

Two basic types of scars may develop depending on the loss or gain of collagen (atrophic and hypertrophic scars). The majority of people with acne scars $(80-90 \%)$ have atrophic scars compared to a minority who show hypertrophic scars and keloids ${ }^{[4]}$.

Atrophic scars are classified into ice pick, rolling, and boxcar types; however, different types of scars can be seen on the same person. Ice pick represents $60-70 \%$ of atrophic scars, rolling type $15-25 \%$, and boxcar type $20-30 \%{ }^{[5]}$.

Ice pick scars are small, dotted, and deep scars $(2 \mathrm{~mm})$ in their depth, in the form of the letter V. Rolling scars are wide (up to $5 \mathrm{~mm}$ ) with a sloping edge and deeper, they reach to the subcutaneous tissue, in the form of the letter M. Boxcar scars are broad depressions $(1.5-4 \mathrm{~mm})$ with sharply defined vertical edges and broad base, it takes the shape of the letter $\mathrm{U}^{[6,7] \text {. }}$

Assessment of the disease load, global severity, and lesion morphology was made using Goodman and Baron Scale, the global acne scarring grading system ${ }^{[8]}$.

\section{Fractional Carbon Dioxide (CO2) laser}

Laser resurfacing using fractional $\mathrm{CO} 2$ laser is considered the most effective modality with satisfactory results ${ }^{[0]}$. It acts by treating the only column of the affected skin leaving intervening areas of skin untreated. The untreated areas help in rapid re-epithelisation of the skin, minimizing the chances of prolonged and serious adverse effects ${ }^{[10]}$. Fractional lasers are classified into either non-ablative 1550-nm erbium-glass or ablative 10600-nm carbon dioxide fractional lasers. Although the former is claimed to be safe, the latter is more effective in treating facial imperfections with better patient satisfaction [11]. Fractional $\mathrm{CO} 2$ laser with high fluence and low density has been used to treat acne scars with varying degrees of success ${ }^{[12,13]}$ The only disadvantage is post-inflammatory hyperpigmentation.

\section{Microneedling Fractional Radiofrequency (RF)}

Radiofrequency is electromagnetic radiation with a frequency range between 3 and $300 \mathrm{GHz}$. With the fractional bipolar RF device, the RF current flows through the skin between the electrode-pin rows. It delivers energy that creates dermal microscopic wounds at predetermined depths, without affecting the epidermis. The energy interspersed between the intact tissue where it elicits a wound healing response, stimulating the new dermal collagen. Fractional RF is considered less invasive than FCO2 laser and has the advantage of creating fewer side effects with a shorter downtime ${ }^{[14,15]}$. The present study aimed to compare the efficacy of fractional $\mathrm{CO} 2$ laser and microneedling fractional RF in treating atrophic acne scars and to study their side effects.

\section{Patients and methods:}

This was a prospective, interventional comparative study conducted on 64 patients with post-acne scars attending the dermatology private center, in Al Diwaniyah, Iraq, during the period from (April 2017-March 2020). Written consent was obtained from all participants. Approval was taken from the local ethical committee before starting the study.

\section{Inclusion criteria:}

. Patients with acne scars meet the grading of 3 and 4 of the Goodman and Baron grading scale.

. Patient with no personal or family history of keloids.

.Patients above 18 years. 
Patient willing to the treatment and they are with a realistic expectation

Exclusion criteria:

.Tendency for keloid formation.

. Receiving isotretinoin therapy for the last six months

. Active infection at the treated area.

Bleeding diathesis and those who have taken anticoagulant therapy

- A serious health problem causing delayed wound healing such as diabetes mellitus, immunocompromised, and those on corticosteroid therapy.

\section{The procedure}

The patients were divided into two groups of 32 each. Group A was treated with fractional (CO2) every six weeks for four sittings (baseline, 6wks, $12 \mathrm{wks}, 18 \mathrm{wks}$ ) and group $B$ was treated with micro-needling fractional (RF) with the same previous schedule. Each patient underwent a subcision of the scars before the irradiation. Subcision was done using 18 gauge cannula in forward-backward and fanning manners.

Topical numbing cream was applied to the treated area for 30 minutes before the procedure.

Treatment parameters were adjusted according to the site of the scars and tolerability of the patient. For group A, laser beams of (120 and $300 \mathrm{~nm}, 50$ and $30 \mathrm{mj}, 100$ spots/ density, with static and dynamic modes) were applied using eCO2 ${ }^{\circledR}$ Laser; Lutronic, Inc., Goyang, South Korea). While in group $\mathrm{B}$, two passes of different parameters of ( intensity $30-50 \%$, RF pulse 150 $200 \mathrm{~ms}$, depth $2-3 \mathrm{~mm}$, single mode, with delay time 250-400 ms) were applied using (Secret ${ }^{\circledR}$; llooda Inc., Suwon, South Korea). The treatment was given every 6 weeks for a total of four sittings. Any patient who satisfied with the result after the $2^{\text {nd }}$ or $3^{\text {rd }}$ session, the next sessions were not performed. The patients were instructed to avoid sun exposure for the next 5 days after each treatment. Topical antibiotic cream with formulation containing sesame oil and honey wax was prescribed over this period.
An objective score was calculated at baseline using Goodman and Baron Scale, and then every visit to evaluate the outcome during treatment. The last evaluation was done 3 months after completion of therapy. Digital photographs were taken at every follow up visit. The response to the treatment was labeled as follows; $0-25 \%$ poor response, 26-50\% fair response, $51-75 \%$ good response, and $76-100 \%$ excellent response.

Data were collected and analyzed using SPSS version 26 where tests, descriptive statistics, frequencies and percentages, and independent sample t-test verified.

\section{Results:}

A total of 64 patients with acne scars were included in this study. The patients were randomly assigned into two groups of 32 patients each. In group A (CO2), 18 (56.25\%) were females and $14(43.75 \%)$ were males, and the majority of them (87.5\%) were in the age group of 21-30 years. On the contrary, only four patients (12.5\%) were in the age group of 31-40 years.

In the case of fractional RF treatment (group B), $21(65.62 \%)$ were females and 11 (34.37\%) were males. The majority $(75 \%)$ of them were between the age of $21-30$ years and the other $25 \%$ between the ages of 31-40 years. Ice peck scars were formed $50 \%$ of all lesions, followed by boxcar and rolling types in the proportion of $30 \%$ and $20 \%$, respectively.

At the baseline point time, out of 32 patients in group A, 18 patients were classified under grade 4 of Goodman and Baron Scale, and 10 patients had grade 3 . The other four patients drop out of the study. Out of the 32 patients treated with fractional RF (group B), 12 classified under grade 4 , and 14 patients had grade 3 . (Table 1)

\section{Objective assessment}

The baseline mean score for group A was 3.64 \pm SD 0.487 , which was reduced to $1.28 \pm \mathrm{DS}$ 0.534 after treatment. The reduction percentage was found to be $64.8 \%$. The baseline mean score for group B was $3.62 \pm 0.491$, which was 
Nabeel K. Al-Hamzawi., AJODRR, 2021 4:44

reduced to $2.15 \pm 0.766$. The reduction changes between the two groups was found to percentage was $40.6 \%$. The difference in be highly significant, $P<0.01$. (Table 2).

Table 1 Characteristics of lesions in both $\mathrm{CO} 2(\mathrm{~A})$ and $\mathrm{RF}(\mathrm{B})$ groups

\begin{tabular}{|c|c|c|c|c|c|c|}
\hline Variable & Grade 2 & Grade 3 & grade 4 & ice peck\% & boxcar \% & rolling \% \\
\hline Group A & 0 & 10 & 18 & 30 & 30 & 40 \\
\hline Group B & 6 & 14 & 12 & 50 & 30 & 20 \\
\hline
\end{tabular}

Table 2 Difference in percent reduction of the mean score between CO2 and RF group

\begin{tabular}{|c|c|c|c|}
\hline Type of therapy & baseline mean score & follow up mean score $\%$ & reduction \\
\hline FCO2 & 3.64 & 1,28 & 64.8 \\
\hline FRM & 3.62 & 2.15 & 40.6 \\
\hline
\end{tabular}

$P<0.01$

Table 3 Comparison of the response to the treatment between the two groups

\begin{tabular}{|c|c|c|}
\hline Response & CO2 & RF \\
\hline Poor & 2 & 8 \\
\hline Fair & 5 & 18 \\
\hline Good & 14 & 6 \\
\hline Excellent & 7 & 0 \\
\hline Total & 28 & 32 \\
\hline
\end{tabular}

Table 4 Percent reduction in different types of scars after treatment

\begin{tabular}{|c|c|c|c|}
\hline Treatment & Ice peck \% Reduction & rolling\% reduction & boxcar\% reduction \\
\hline FCO2 & $40 \%$ & $70 \%$ & $60 \%$ \\
\hline FRM & 0 & $50 \%$ & $40 \%$ \\
\hline
\end{tabular}

In group $A(\mathrm{CO} 2), 02$ patients showed a poor response (0-25\% improvement in point score), the majority of patients 14 had good response i.e. $51-75 \%$ improvement in point score, 05 patients achieved a fair response, and 07 patients had an excellent response [Figure 1, 2].

In group $\mathrm{B}(\mathrm{RF}), 08$ patients had a poor response (0-25\% improvement in point score), the majority of patients 18 achieved fair response (26-50\%), 06 patients had a good response, and no one categorized under excellent results [Figure 3] (Table 3)
Scarring intensity in group A reduced as follows: rolling and boxcar scars showed $70 \%$ and $60 \%$ reduction in point score respectively, while ice peck showed a $40 \%$ reduction in point score. Whereas, in group B scarring intensity reduced by $50 \%$ for rolling scars, $40 \%$ for boxcar, and no changes were recorded for ice peck scars. (Table 4)

Adverse reactions include erythema, local edema, and hyperpigmentation. Erythema and edema were transient and lasted no more than 24 hours in the FRM group, while it extended to AJODRR: https://escipub.com/american-journal-of-dermatological-research-and-reviews/ 4 
5- 10 days in the FCO2 group according to the particularly in patients with type IV Fitzpatrick parameters used. Post-inflammatory hyperpig- skin type [ Figure 4]. No pigmentary changes mentation was recorded in the FCO2 group, were noticed in the RF group.

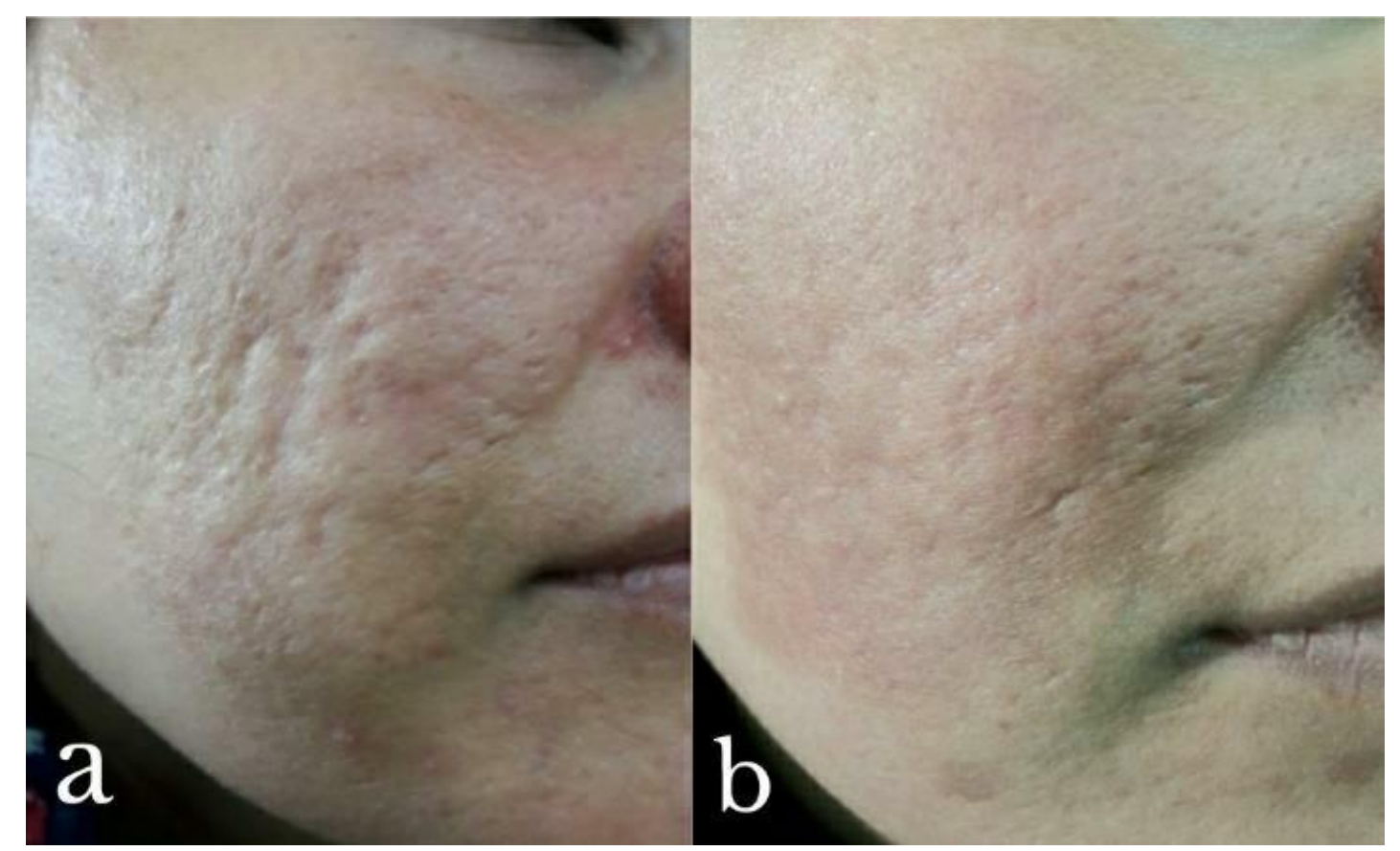

Figure 1: a. Grade 4 post acne scars b. Marked improvement of acne scars from grade 4 to grade 1 after treatment with subcision and fractional $\mathrm{CO} 2$ laser

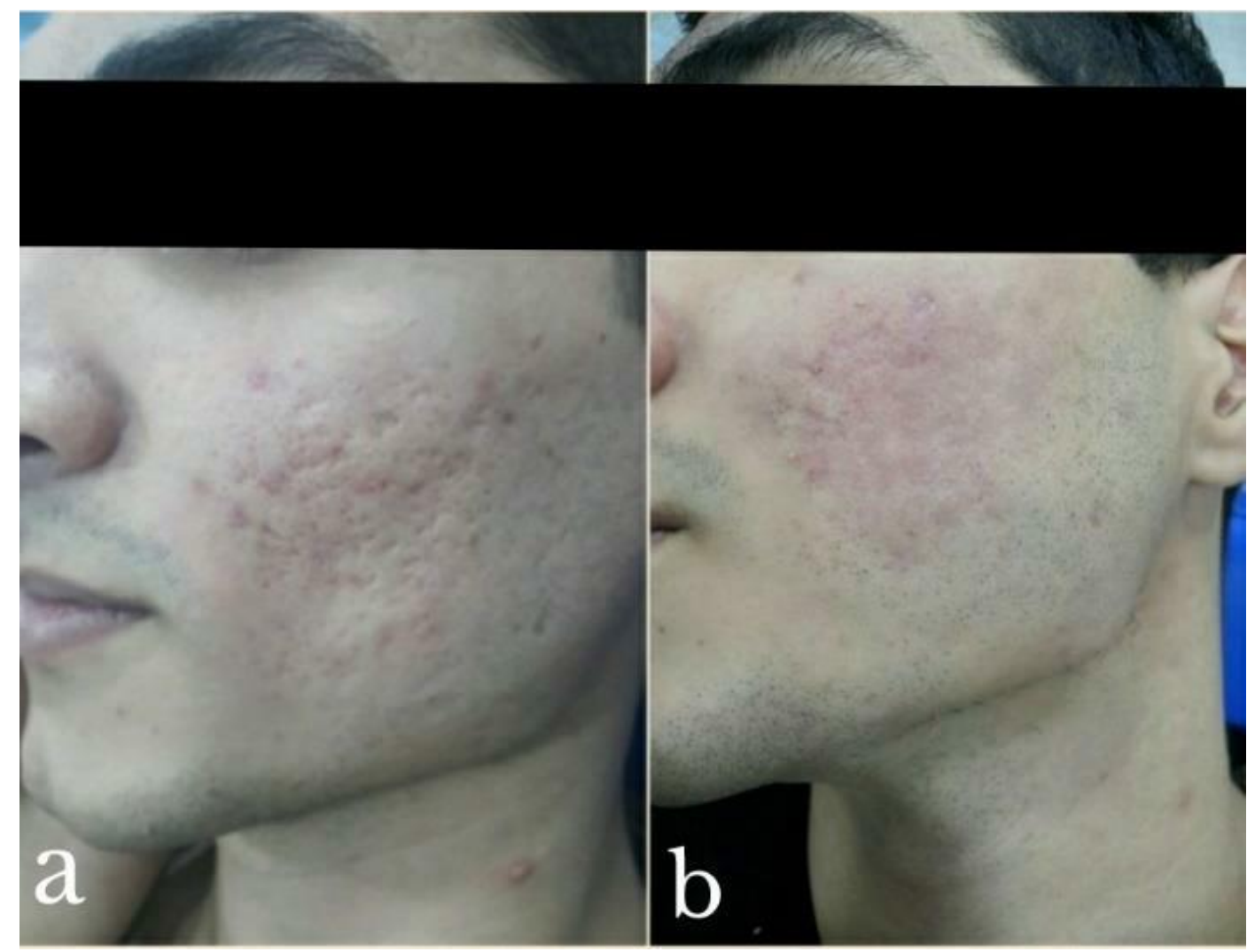

Figure 2: a. Grade 4 acne scars b. Improvement in scarring from grade 4 to grade 2 after treatment with subcision and fractional CO2 laser 


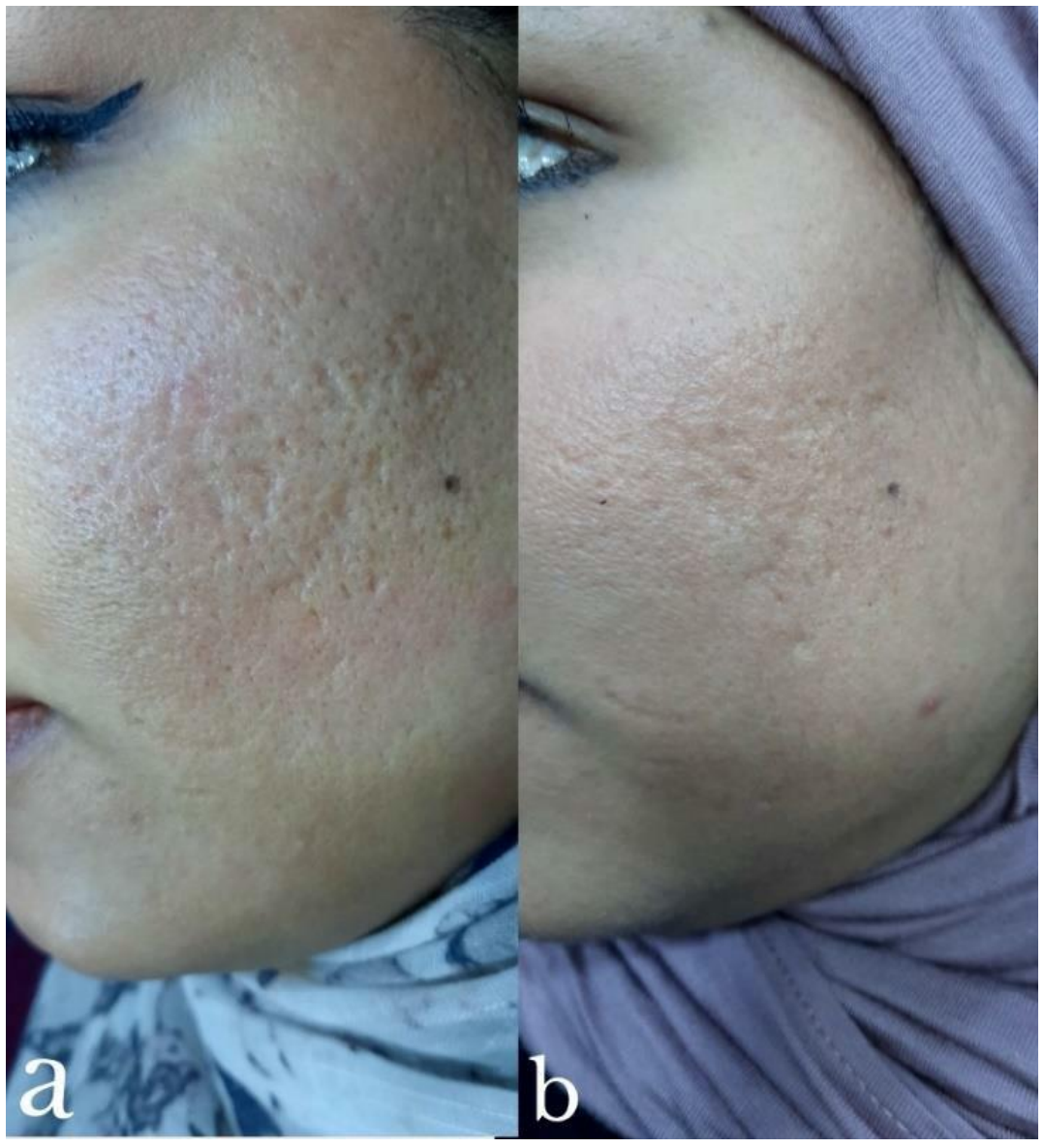

Figure 3: a. Grade 4 post acne scars b. Improvement in scarring from grade 4 to grade 2 after treatment with subcision and microneedling fractional RF

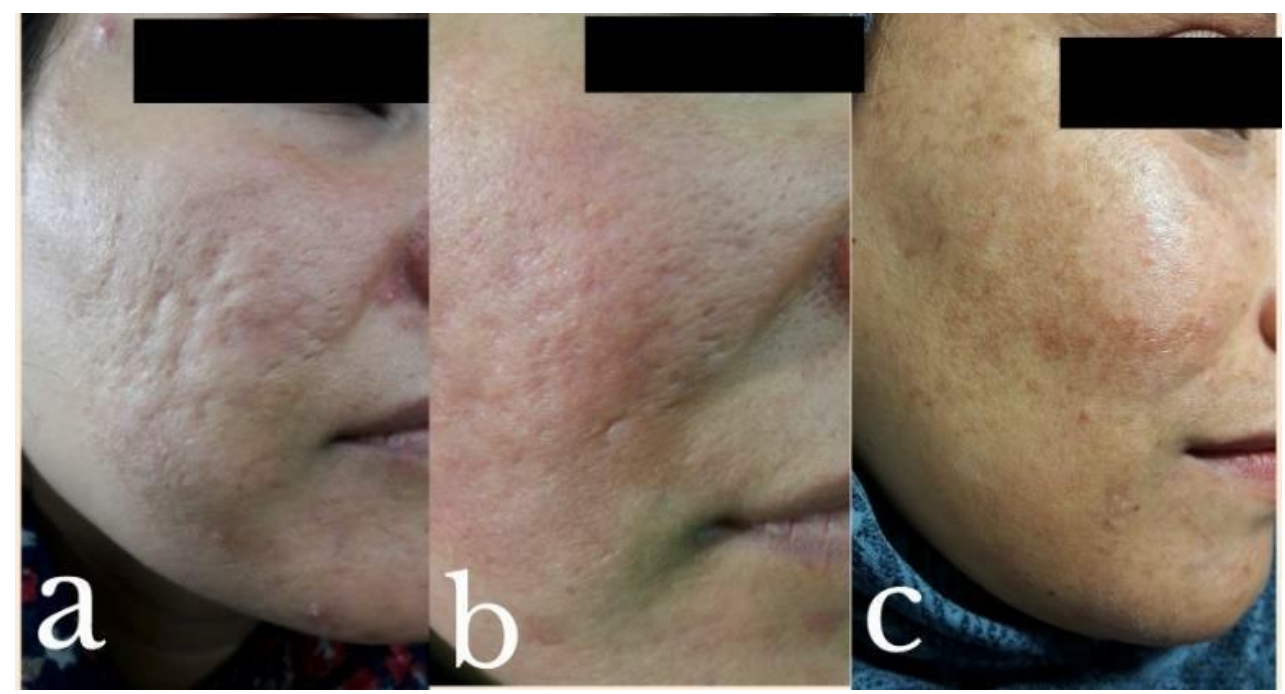

Figure 4 a. Post acne scars treated with fractional $\mathrm{CO} 2$ laser b. obvious erythema 10 days after $\mathrm{CO} 2$ laser treatment $\mathrm{c}$. post-inflammatory hyperpigmentation

\section{Discussion:}

Because of the increasing risk of depression among patients who are most affected by acne scars, aggressive treatment is required to eliminate them. The best treatment option is one that achieves marked improvement in the look, and thus improves the quality of life. In this study, patients were divided into two groups, group A treated with fractional $\mathrm{CO} 2$ laser and group B treated with microneedling fractional 
RFF. Both treatment options were preceded by subcision with each session. Choosing either of the therapeutic options, depend on certain considerations in each patient. These include the skin type of the patient, the type of the scar, the depth of the scar, and its duration. Besides, to the downtime that suits the patient. The majority of patients who were treated with fractional $\mathrm{CO} 2$ laser had Fitzpatrick skin type 3 and their scar duration ranged between 5 to 8 years. Whereas most of the patients who underwent RF had skin type 4 and their scar duration ranged between 2 to 5 years. In each group, variable parameters particularly that related to the fluence and intensity were used in different patients. The variability in parameters was made according to the differences in the type, location, and depth of scars among participants. These principles were adopted to achieve the best outcomes with the less likely risk of post-inflammatory hyperpigmentation. Interestingly, in group A, the main changes were noted in rolling, and boxcar scars, whereas in group B, the main improvement was exhibited in rolling scars. This is explained by the double effect of $\mathrm{CO} 2$, they encourage renewable processes and increase the production of matrix proteins by fibroblasts, which is more beneficial for ice peck type of scars.

The immediate adverse reactions of both modalities include pain and erythema and scab formation. Erythema was more prolonged in a patient treated with $\mathrm{FCO} 2$ and its duration depended on the density and fluence of the applied laser, whereas in the RF group, erythema subsided after 24 hours. Although the depth of penetration of $\mathrm{CO} 2(1.9 \mathrm{~mm})$ is less than RF $(3.5 \mathrm{~mm})$, it is more aggressive and leads to disruption of the cells and damage to the dermoepidermal junction. Therefore, patients who undergo this modality required more downtime to recover from erythema and scab formation. As a consequence, the risk of post-inflammatory pigmentation is more frequent with $\mathrm{FCO} 2$ treatment.

The results showed that the fractional $\mathrm{CO} 2$ laser was more effective and practical than RF in treating acne scars, although it required a longer downtime and may be associated with PIH. The selection of treatment depends on which type of scar is predominant, the grade of the scars, and the skin phenotype

of the patient. Fractional $\mathrm{CO} 2$ modality is more suitable for patients with low phenotype skin, and have grade 4 intensity scarring with rolling and boxcar predominant lesions. While RF is more vulnerable to those with high phenotype skin and has grade 3 or 2 with rolling predominate lesions. Subcision adds a more synergistic effect on the treatment. It is more effective for treating rolling than boxcar scars. It was done before the first session. With ice peck scars, frequent subsicion was needed to be done before each session to achieve acceptable results.

Conclusion: To achieve the best results, treatment should be tailored according to the skin type, depth of the scar, and the length of the downtime to be adopted. Fractional $\mathrm{CO} 2$ laser resurfacing was considered superior over the microneedling fractional RF technique in the treatment of post-acne scars. However, combined treatment is more effective and can shorten the number of sessions required. Boxcar and rolling types of scars showed a high response to treatment than icepick scars

\section{References:}

[1]. Williams C, Layton AM. Persistent acne in women: implications for the patient and therapy. American Journal of Clinical Dermatology. 2006;7(5):281290.

[2]. Capitanio B, Sinagra JL, Bordignon V, Fei PC, Picardo M, Zouboulis CC. Underestimated clinical features of postadolescent acne. Journal of the American Academy of Dermatology. 2010;63(5):782-788.

[3]. Kurokawa I, Danby FW, Ju Q, et al. New developments in our understanding of acne pathogenesis and treatment. Experimental Dermatology. 2009;18(10):821-832.

[4]. Fabbrocini, G., Annunziata, M. C., D'Arco, V., De Vita, V., Lodi, G., Mauriello, M. C., Pastore, F., \& Monfrecola, G. (2010). Acne scars: pathogenesis, classification and treatment. Dermatology research and practice, 2010, 893080. 
[5]. Connolly D, Vu HL, Mariwalla K, Saedi N. Acne Scarring-Pathogenesis, Evaluation, and Treatment Options. The Journal of Clinical and Aesthetic Dermatology. 2017 Sep;10(9):12-23.

[6]. Fabbrocini G, Annunziata MC, D'Arco V, et al. Acne Scars:Pathogenesis, Classification and Treatment. Dermatology Research and Practice. 2010;2010:893080

[7]. Petrov, A., \& Pljakovska, V. (2016). Fractional Carbon Dioxide Laser in Treatment of Acne Scars. Open access Macedonian journal of medical sciences, 4(1), 38-42.

[8]. GOODMAN, GREG J. BARON, JENNIFER A. Scarring, Dermatologic Surgery: December 2006 - Volume 32. - Issue 12 - p 1458-1466

[9]. Gozali MV, Zhou B. Effective treatments of atrophic acne scars. J Clin Aesthet Dermatol. 2015;8(5):33-40.

[10]. Susan W, Brian ST, James C, Gregory A, Andrei E, llya $Y$, et al. Fractional Photothermolysis: A novel aesthetic laser surgery modality. Dermatol Surg. 2007;33:525-34.

[11]. Cho SB, Lee SJ, Cho S, Oh SH, Chung WS, Kang JM, et al. Non-ablative 1550-nm erbium-glass and ablative $10600-\mathrm{nm}$ carbon dioxide fractional lasers for acne scars: A randomized split-face study with blinded response evaluation. J Eur Acad Dermatol Venereol. 2010;24:921-5.

[12]. Alster TS, Tanzi EL, Lazarus M. The use of fractional laser photothermolysis for the treatment of atrophic scars. Dermatol Surg. 2007;33:295-9.

[13]. Gold MH, Heath AD, Biron JA. Clinical evaluation of the SmartSkin fractional laser for the treatment of photodamage and acne scars. J Drugs Dermatol. 2009;8:s4-8.

[14]. Rongsaard N, Rummaneethorn P. Comparison of a fractional bipolar radiofrequency device and a fractional erbium-doped glass 1,550$\mathrm{nm}$ device for the treatment of atrophic acne scars: a randomized split-face clinical study. Dermatol Surg. 2014;40:14-21.

[15]. Gozali MV, Zhou B. Effective treatments of atrophic acne scars. J Clin Aesthet Dermatol. 2015;8(5):33-40. 